A dedicated device for measuring the magnetic field of the ND280 magnet in the T2K experiment

This article has been downloaded from IOPscience. Please scroll down to see the full text article.

2012 JINST 7 P01018

(http://iopscience.iop.org/1748-0221/7/01/P01018)

View the table of contents for this issue, or go to the journal homepage for more

Download details:

IP Address: 137.138.93.221

The article was downloaded on $23 / 07 / 2013$ at $14: 08$

Please note that terms and conditions apply. 


\title{
A dedicated device for measuring the magnetic field of the ND280 magnet in the T2K experiment
}

\section{E. Frank, ${ }^{a}{ }^{a}$ A. Ariga, ${ }^{a}$ T. Ariga, ${ }^{a}$ F. Bay, ${ }^{a}$ F. Bergsma,${ }^{b}$ A. Ereditato,${ }^{a}$ F. Garnier,${ }^{b}$ P.-A. Giudici, ${ }^{b}$ F. Hahn, ${ }^{b}$ A. Kehrli, ${ }^{b}$ I. Kreslo, ${ }^{a}$ M. Losasso,,${ }^{b, c, 2}$ M. Messina ${ }^{a}$ and S. Rangod ${ }^{b}$}

\author{
${ }^{a}$ Albert Einstein Center for Fundamental Physics, Laboratory for High Energy Physics (LHEP), \\ University of Bern, \\ Sidlerstrasse 5, CH-3012 Bern, Switzerland \\ ${ }^{b}$ European Organization for Nuclear Research (CERN), \\ CH-1211 Geneva 23, Switzerland \\ ${ }^{c}$ Fusion for Energy, \\ c/ Josep Pla 2, 08019 Barcelona, Spain \\ E-mail: eike.frank@lhep.unibe.ch
}

ABSTRACT: This paper describes a dedicated device to map the magnetic field of the T2K near detector (ND280) magnet, which runs at a nominal field value of $0.2 \mathrm{~T}$, with an accuracy of the order of a Gauss. A high accuracy mapping is a key ingredient in order to provide the required momentum accuracy in the ND280 time projection chambers (TPCs) allowing T2K to measure precisely the PMNS matrix parameters $\Delta m_{23}^{2}$ and $\theta_{23}$. This paper describes the design and realization of the device as well as its performance during operation. The reported results show that the aimed at goals are reached.

KEYWORDS: T2K experiment; ND280 magnet; Measurement of magnetic field; Hall probe calibration; Magnetic field mapping

\footnotetext{
${ }^{1}$ Corresponding author.

${ }^{2}$ Now at Fusion for Energy, Barcelona, Spain.
} 


\section{Contents}

1 Introduction 1

2 The mapping device 2

2.1 Mechanics and electronics 2

2.2 Device survey 4

2.3 Hall probe calibration 6

3 Calibration data, equalization and alignment corrections 9

4 Conclusions 11

\section{Introduction}

T2K $[1,2]$ is the first long-baseline neutrino beam experiment which uses an intense off-axis neutrino beam with the goal of determining the unknown parameter $\theta_{13}$ [3] of the PMNS neutrino mixing matrix [4]. T2K also aims to precisely measure the parameters $\Delta m_{23}^{2}$ and $\theta_{23}$ with an improvement of an order of magnitude on the current sensitivities. This is obtained from the comparison of the measured fluxes at a near detector station, $280 \mathrm{~m}$ away from the neutrino source (ND280), and at Super-Kamiokande, a 50 kton water Čerenkov detector, $295 \mathrm{~km}$ away. To achieve the aimed precision, a good knowledge of the neutrino flux and energy spectrum is essential, which can be obtained from the ND280 near detector station.

The magnetic field for ND280 is generated by the former UA1/NOMAD dipole magnet $[5,6]$. This magnet (figure 1) was refurbished at CERN for the purpose of the T2K experiment and shipped in 2008 to the Japan Proton Accelerator Research Complex (J-PARC), situated in Tokai. The magnet has a weight of $\sim 900$ tons and its inner dimensions are $3.6 \mathrm{~m} \times 3.5 \mathrm{~m} \times 7.0 \mathrm{~m}$. The magnet consists of $16 \mathrm{C}$-shaped yokes and 4 coils made of 26 double "pancakes" for a total of 208 turns, generating a dipole magnetic field of $0.2 \mathrm{~T}$ for the purpose of $\mathrm{T} 2 \mathrm{~K}$. Inside the coils the so-called basket structure is placed. This support frame is made out of stainless steel with inner dimensions of $2.3 \mathrm{~m} \times 2.4 \mathrm{~m} \times 6.6 \mathrm{~m}$ and holds most of the sub-detectors of ND280, as is shown in figure 2 . Most upstream is the $\pi^{0}$-detector (P0D), built mainly of scintillators interlaced with water, followed by the tracker which contains three time projection chambers (TPCs) and two fine-grained detectors (FGDs) in a sandwich structure. The downstream electromagnetic calorimeter (Downstream ECal) completes the detectors inside the basket. The basket is surrounded by further electromagnetic calorimeters and by the muon range detectors (SMRDs) which instrument the magnet yoke.

The tracker is designed to study charged current neutrino interactions occurring in the FGDs and other detector parts. Each of the three rectangular TPC chambers has an outer dimension of $2.3 \mathrm{~m} \times 2.4 \mathrm{~m} \times 1.0 \mathrm{~m}$ and an inner sampling length of $700 \mathrm{~mm}$. Each TPC consists of an inner box that holds an argon-based drift gas within an outer box that holds $\mathrm{CO}_{2}$ as insulating gas. Copper strips of precisely $11.5 \mathrm{~mm}$ pitch in conjunction with a central cathode panel produce a uniform 


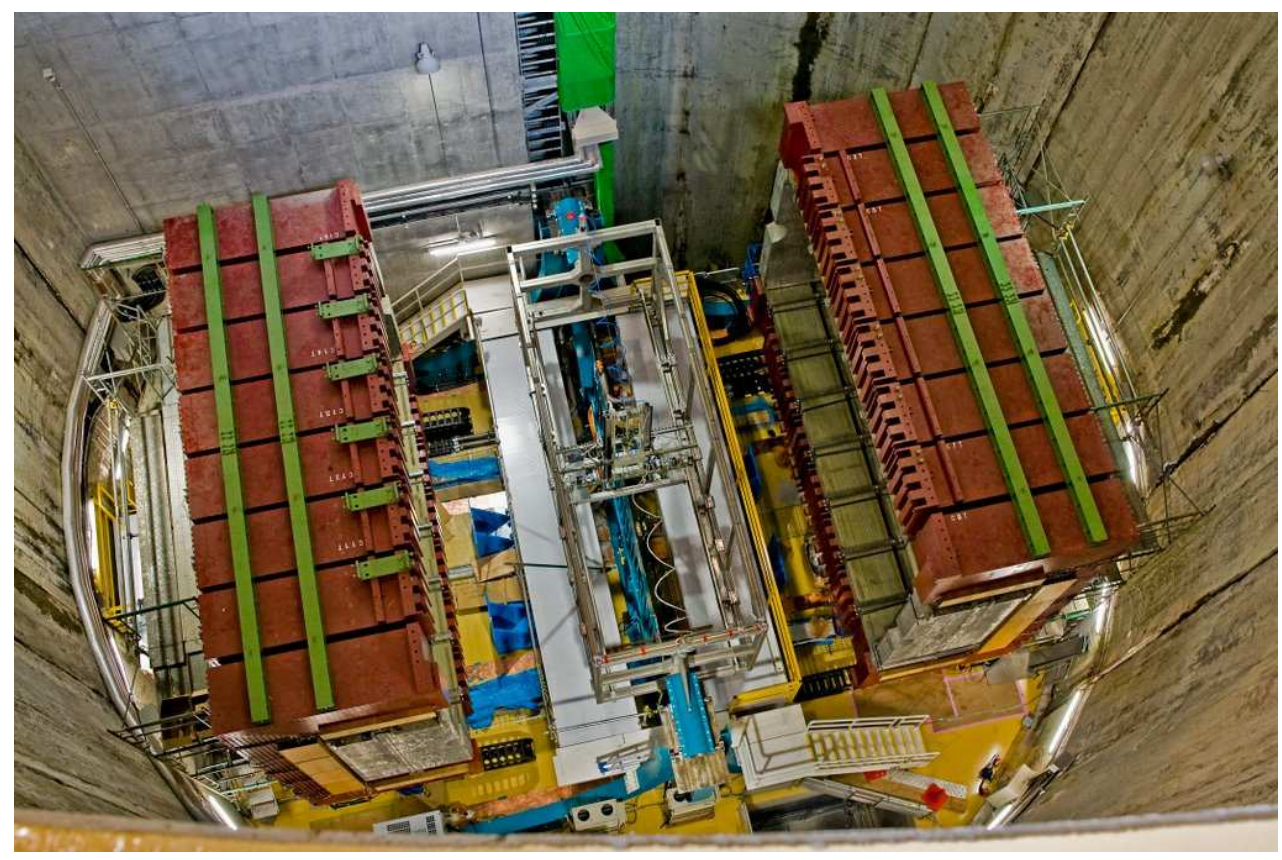

Figure 1. Top view of the open T2K ND280 magnet with the mapping device installed.

electric drift field, roughly aligned with the magnetic field. Bulk micromegas detectors are used for the gas-amplified readout of ionization electrons with an anode pad segmentation of $70 \mathrm{~mm}^{2}$. Each TPC has two readout planes with twelve micromegas modules for a total of 72 modules. The signal pattern in combination with the arrival time allows a 3D track reconstruction of charged particles. More details on the design and performance of the TPCs can be found in [7].

The precise mapping of the magnetic field in the instrumented region of the ND280 detector complex has several goals. The magnetic field itself is a key element for precisely measuring the momentum of the charged particles passing through the inner detector volume. The performance requirement of the TPCs corresponds to a momentum resolution of better than $10 \%$ and to a momentum scale known to better than $2 \%$. This can be achieved by measuring the magnetic field with an accuracy of the order of a Gauss in the directions perpendicular to the drift direction of the electrons in the TPCs. These magnetic field components distort the drift of the electrons in the TPC gas volume.

Besides distortions, it is also important to know the absolute scale of the measured magnetic field values. This is guaranteed by a careful calibration of the Hall probes as described in section 2.3. More details on the construction and operation of the device can be found in [8]. An additional publication is being prepared to show results from the full measurement campaign [9].

\section{The mapping device}

\subsection{Mechanics and electronics}

The coordinate reference system used for the mapping device is the same as that of the ND280 detector (figure 2). The $\mathrm{x}$-axis is parallel to the main component of the magnetic field, the $\mathrm{y}$-axis is 


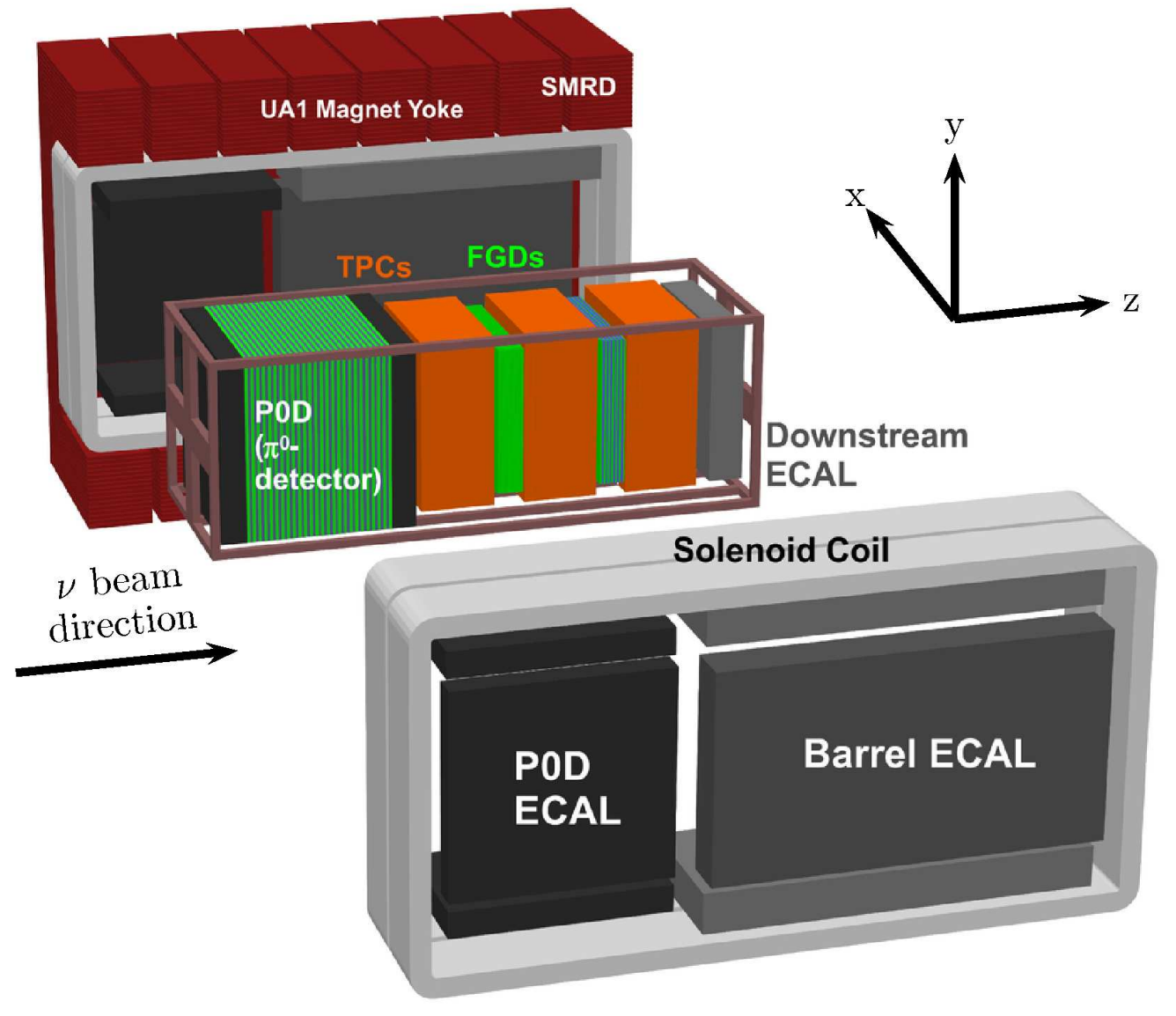

Figure 2. The ND280 detectors in the magnet with magnet outer dimensions of $5.6 \mathrm{~m} \times 6.1 \mathrm{~m} \times 7.6 \mathrm{~m}$.

antiparallel to the line of gravity, and the orthogonal z-axis is in the direction of the neutrino beam. However, small deviations of the order of a mrad exist and were determined by a series of surveys (see section 2.2).

The mapping device was built by the CERN PH-DT group and the T2K Bern group. It was designed to measure a volume slightly larger than the instrumented region inside the basket. The device has a total weight of $\sim 700 \mathrm{~kg}$ and consists of three parallel arms. Two long arms cover the width (x-direction) of the detector region $(2.2 \mathrm{~m})$ and are movable in y $(2 \mathrm{~m}$ in height) and $\mathrm{z}$ (6 $\mathrm{m}$ in length), and a shorter third arm of $1.8 \mathrm{~m}$ length (x-direction) is used to crosscheck the measurements at lower positions in y-direction. Movement in the z-direction required additional rails to be temporarily installed into the basket which were aligned with the mapping device.

The whole equipment was built of non-magnetic materials as aluminum and stainless steel to prevent undesired field distortions. The device can be moved by means of three pneumatic motors (one for the movement in y-direction and two for the movement along the z-axis). A drawing and a photograph of the device are shown in figures 3 and 4 , respectively.

The position of the mapping device during its operation is read out by four optical encoders (two for the y-and two for the z-direction) which allow the user to control the movement of the equipment. The encoder resolution of $10 \mu \mathrm{m}$ allows knowledge of the actual position of the mea- 


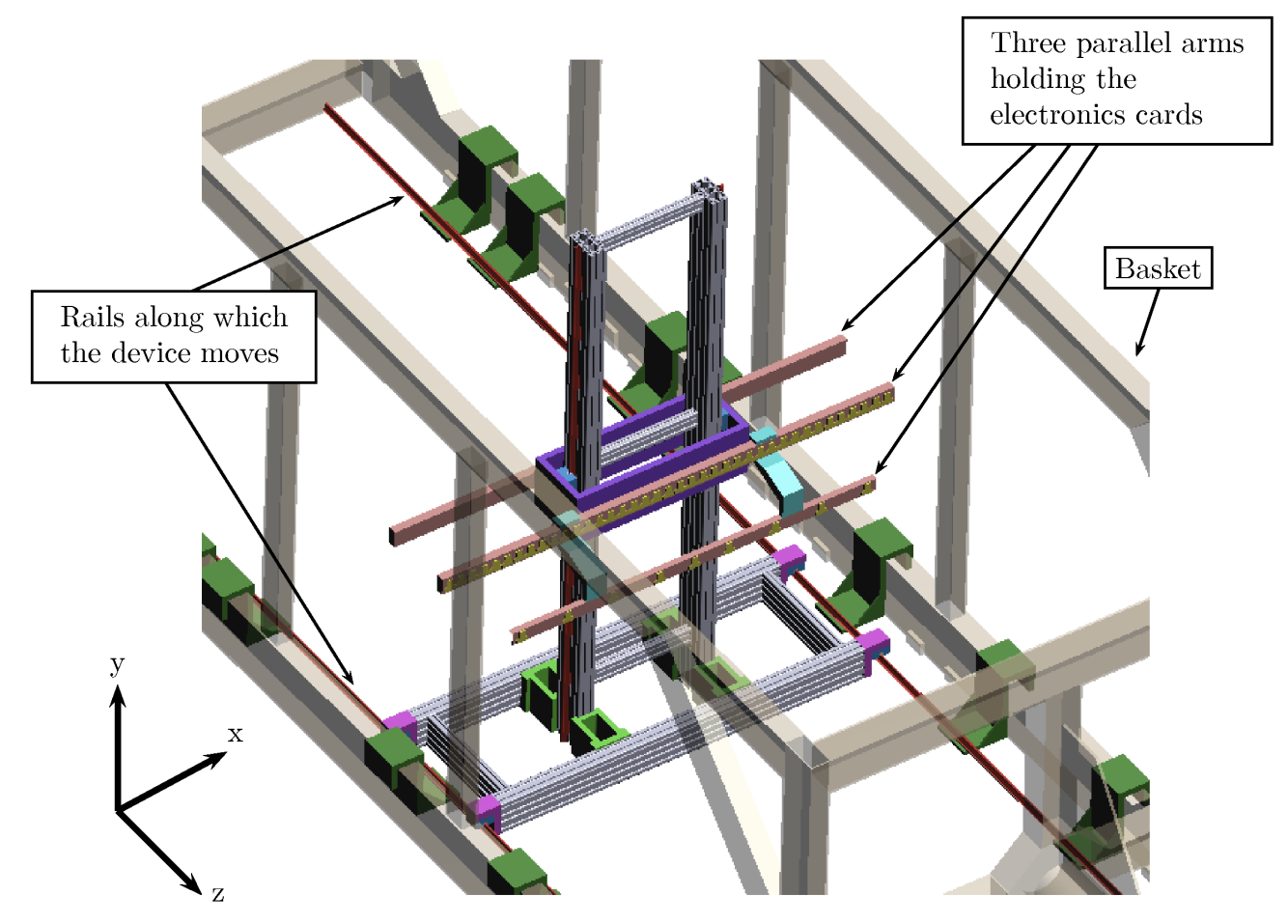

Figure 3. Drawing of the mapping device installed into the basket.

surement bench at the order of $0.1 \mathrm{~mm} .^{1}$ This is an order of magnitude better than the required precision of $\sim 1 \mathrm{~mm}$.

In total 89 electronics cards [10] are installed onto the three arms of the device. Each card is equipped with three Hall probes, which measure the voltage induced by the Hall effect for each component of the magnetic field $\left(B_{x}, B_{y}\right.$, and $\left.B_{z}\right)$, and also contains a temperature sensor as well as the necessary readout electronics (figure 5). The 89 electronics cards are distributed over four readout chains. The user can communicate with the cards via CAN-Bus [11] to activate the routines for the initialization and movement of the device and for data readout. The data of the three Hall probes and the temperature sensor are individually read out and stored. The total readout time per card is $270 \mathrm{~ms}$.

Each of the two longer arms of the device holds 39 cards covering a range of $2166 \pm 1 \mathrm{~mm}$ with a distance of $57 \pm 0.2 \mathrm{~mm}$ from center-to-center of each card in the x-direction. The arms are separated in z-direction by $383 \mathrm{~mm}$. An additional parallel third arm holds 11 cards spread over $1710 \pm 1 \mathrm{~mm}$. With respect to the second arm, the third arm is installed at a distance of $201 \mathrm{~mm}$ in z-direction and $255 \mathrm{~mm}$ lower (y-direction).

\subsection{Device survey}

The positioning and angular deviations from the desired axes were obtained from several surveys [12] of:

\footnotetext{
${ }^{1}$ Thermal expansion of the material is negligible as the temperature fluctuations during the operation of the magnet are below two degrees.
} 


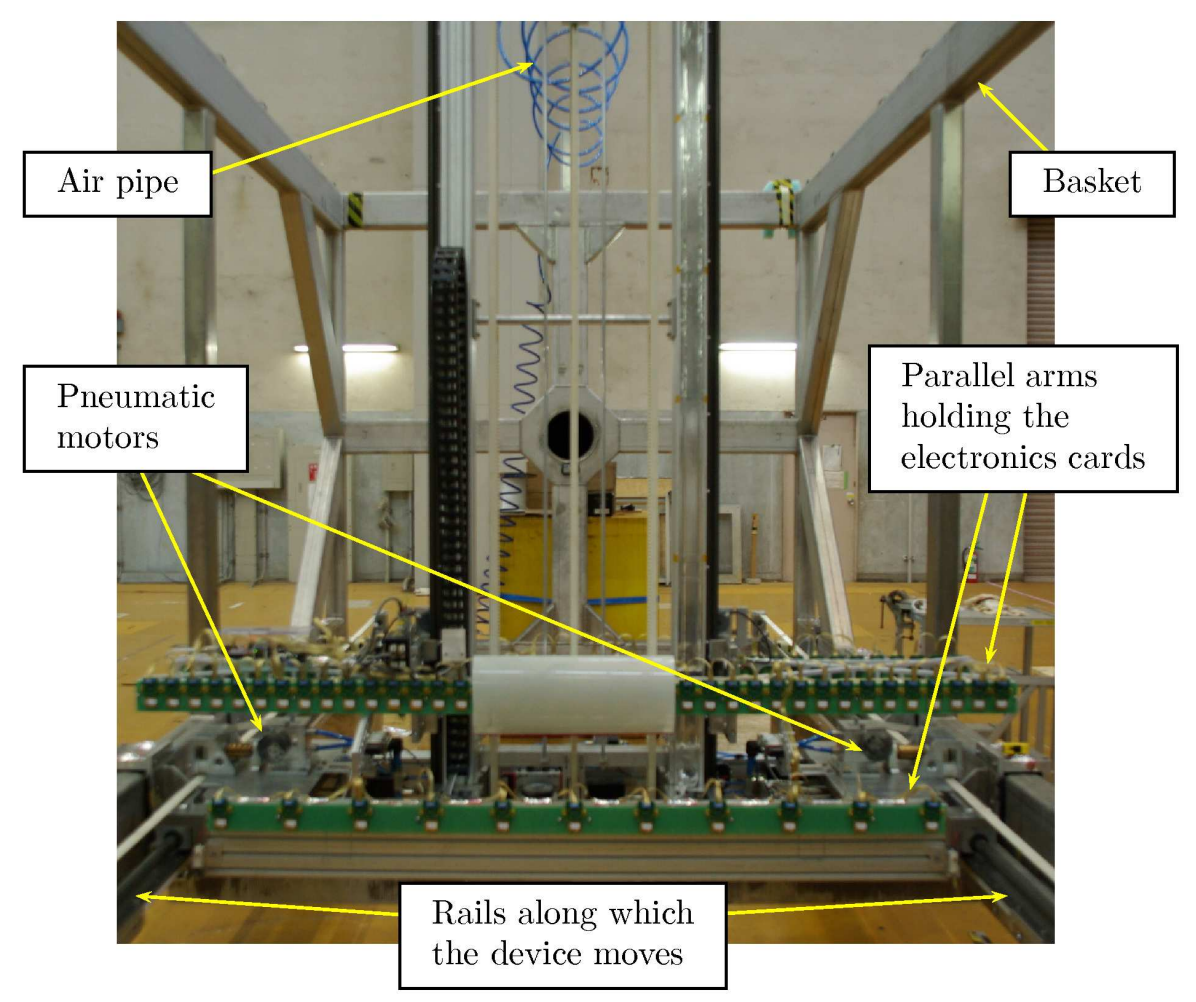

Figure 4. The mapping device in the basket before installation into the ND280 magnet.

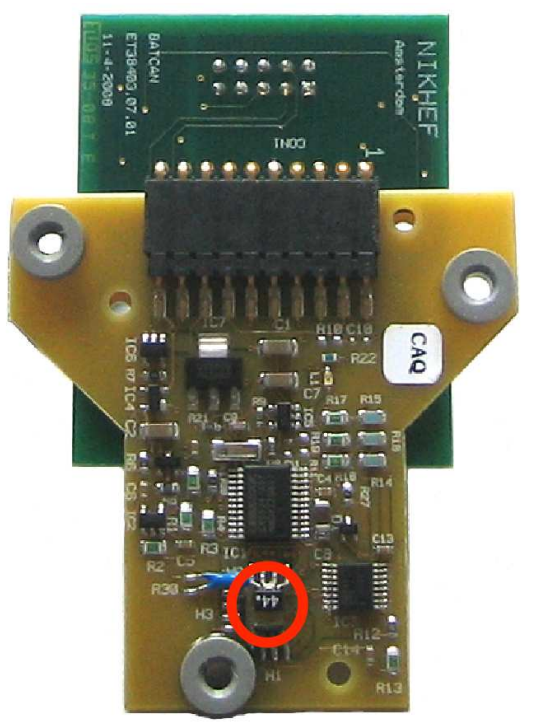

Figure 5. One of the cards with its readout electronics is shown. The red circle in the picture indicates the three orthogonal Hall probes which are placed on a small glass cube. 


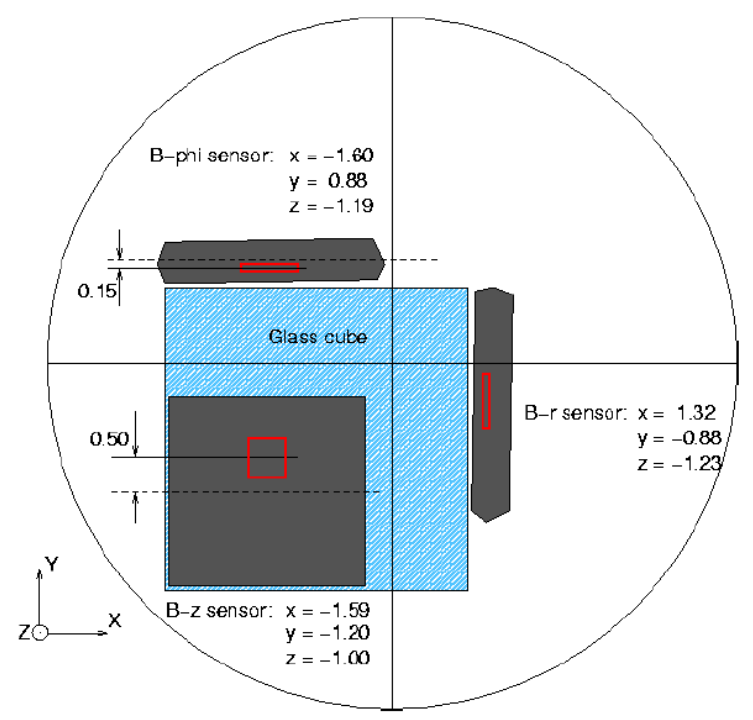

Figure 6. Displacements of the Hall probes with respect to each other in $\mathrm{mm}$ on the glass cube holding the three Hall probes.

1. the mapping equipment and rails with respect to the basket at CERN.

2. the device with respect to the general ND280 reference frame before the actual measurements.

3. the device after the field measurement campaigns.

4. the positions of the individual Hall probes on the electronics cards.

The x-axis of the ND280 reference frame was chosen to coincide with the corresponding axis of the coordinate system of the mapping device. The second of the above mentioned surveys showed that the $y$-axis of the mapping device was rotated by $0.8 \mathrm{mrad}$ with respect to the $y$-axis of the ND280 reference frame. The center of the magnet is the origin of the mapping device reference frame as well as of the ND280 coordinate system.

The three Hall probes of each card are displaced relative to each other as can be seen in figure 6. The measured voltages were associated to the point calculated as the center of mass of the three probes. This method induces an error on the position of less than $1 \mathrm{~mm}$. This is negligible, since the magnetic field changes with less than 2 Gauss per $\mathrm{cm}$ in the region with the strongest field variations.

\subsection{Hall probe calibration}

For the Hall probe calibration, which was performed at CERN [13], each card was placed in a highly uniform field whose strength was monitored by an NMR probe with a precision of better than 1 Gauss. This setup was originally designed for the calibration of the field mapping equipment of the LHC ATLAS experiment $[14,15]$. Pictures of the setup are shown in figures 7 and 8 . Each card was turned to many different orientations with polar angle $\theta$ and azimuth angle $\phi$ that were 


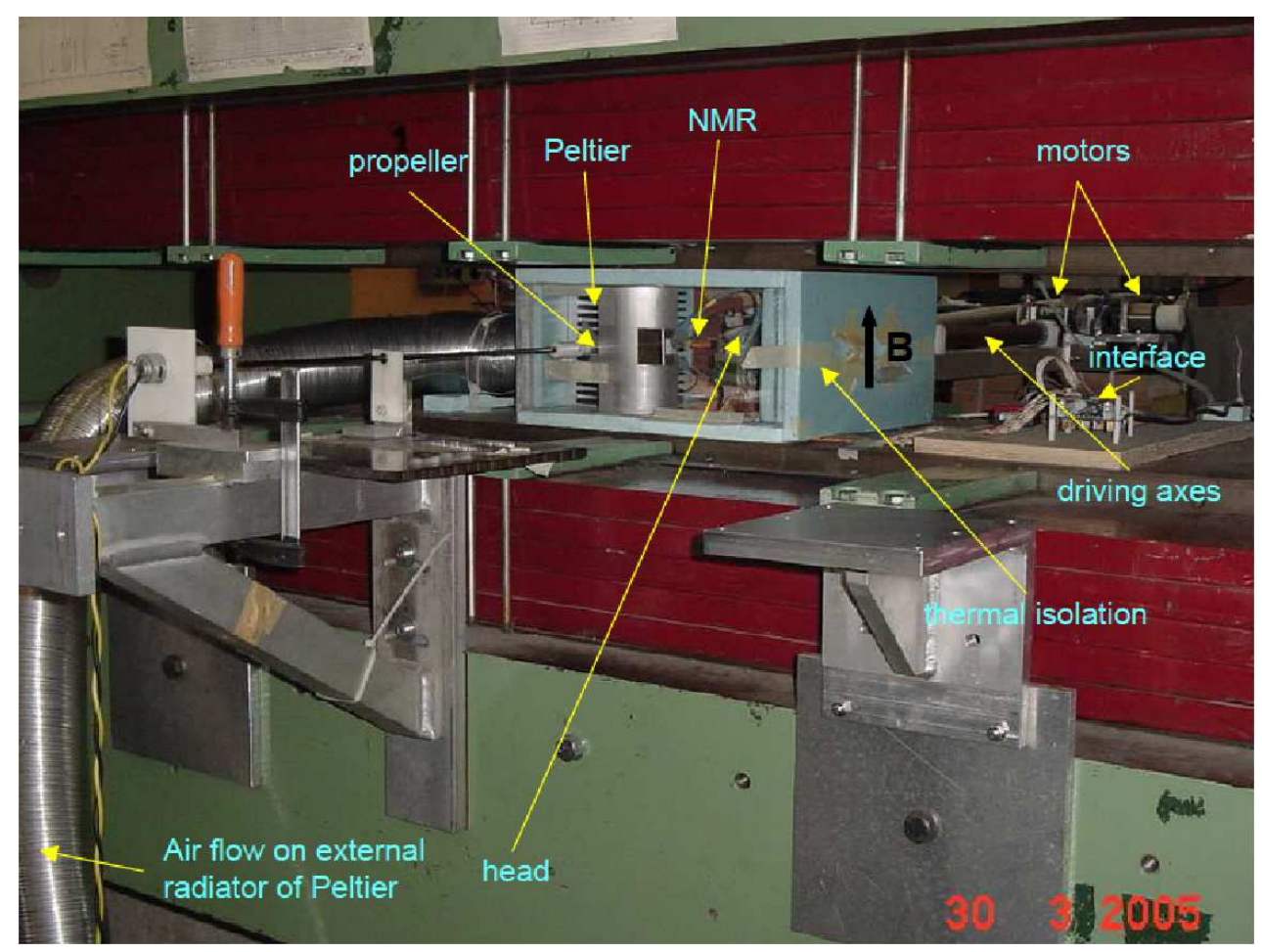

Figure 7. Photograph of the calibration setup. In a homogenous magnetic field (directed bottom to top) a thermally isolated box is mounted which is temperature controlled by a Peltier element and a ventilator. Inside the box an NMR probe monitors the magnetic field and the head to hold the cards with the Hall probes is installed. The head can be rotated freely in three dimensions, which is done by two external motors via driving axes.

precisely measured by three orthogonal pickup coils. The pickup coils rotate together with the card and monitor the change in the effective magnetic field, from which the rotational angle can be inferred. The measurements were repeated for several field strengths and temperatures. The Hall voltage (V) is decomposed into orthogonal functions. Spherical harmonics $Y$ are used for $\theta$ and $\phi$ and Tschebyshev polynomials $T$ for the modulus of the field $\mathrm{B}$ and temperature $\mathrm{t}$ :

$$
V(B, t, \theta, \phi)=\sum_{k} \sum_{n} \sum_{l} \sum_{m=0}^{l} c_{k l m} T_{k}(B) d_{n l m} T_{n}(t) Y_{l m}(\theta, \phi)
$$

Using this series, a total of about 200 calibration parameters was calculated for each probe. A separate angular calibration was used to find the orientation of the calibrated coordinate system relative to three feet that support the card on the mapping device.

All Hall probes were calibrated at $0.2 \mathrm{~T}$ and $1.14 \mathrm{~T}$, and the probes which deviated more than 2 Gauss at a field of $0.2 \mathrm{~T}$ were rejected. A few probes were also calibrated at $0.1 \mathrm{~T}$ as a cross-check, the results were found to be consistent. Figure 9 shows the measurements for one of the probes. The accuracy for the angular alignment between the three probes on a electronic card was measured to be $\pm 2 \mathrm{mrad}$. An improvement on both values, the accuracy of the magnetic field and the angular alignment, can be obtained from the actual mapping data, as described in $[8,9]$. All probes have an 


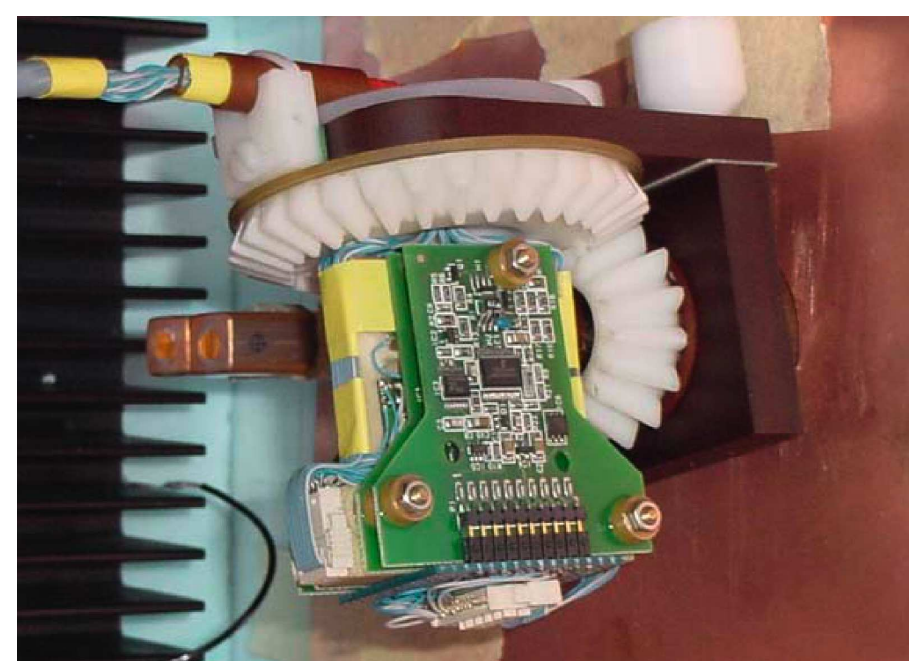

Figure 8. Photograph of one of the electronics cards mounted onto the calibration setup.
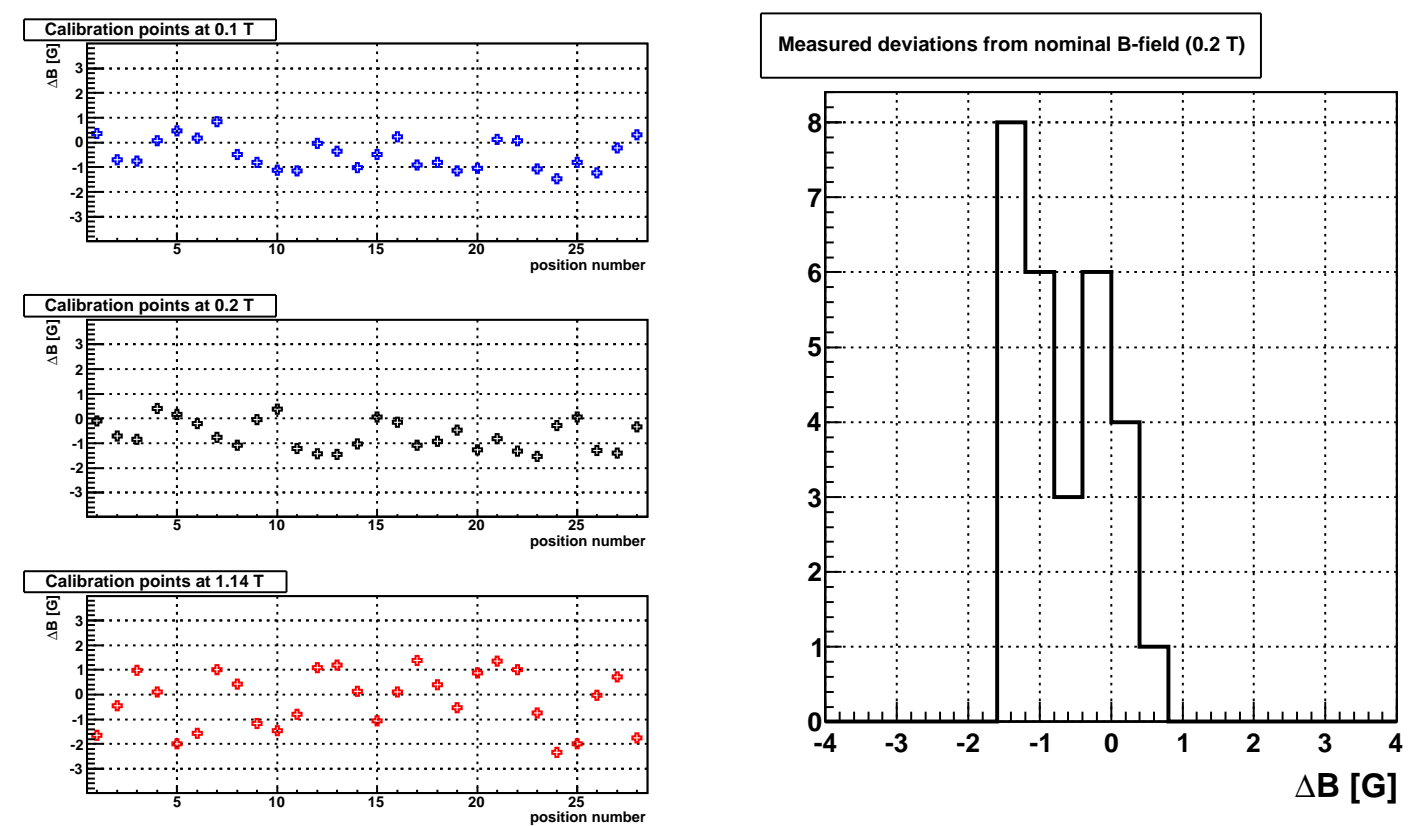

Figure 9. Calibration measurements for one of the Hall probes at 28 angles in $45^{\circ}$ steps. The measurements for three different magnetic field values are shown on the left (top: $0.1 \mathrm{~T}$, middle: $0.2 \mathrm{~T}$, bottom: $1.14 \mathrm{~T}$ ). At a magnetic field of $0.2 \mathrm{~T}$ the deviation of $\mathrm{B}$ from the nominal magnetic field is below 2 Gauss for all measured angles, as can be inferred from the distribution on the right.

intrinsic resolution of 0.2 Gauss, which was obtained from repeated B-field measurements under identical conditions, constant B-field, temperature and angle with respect to the magnetic field. 


\section{Calibration data, equalization and alignment corrections}

To calibrate the mapping device as a whole, a dedicated run in the ND280 magnet was performed, during the measurement no other detectors were installed in the basket. A ramping cycle was done with $250 \mathrm{~A}$ steps from $0 \mathrm{~A}$ to $1000 \mathrm{~A}$, and measurements were taken at points on a rectangular grid through the volume to be instrumented with ND280 detectors (see figure 2). The device was moved in steps of $10 \mathrm{~cm}$ along the beam direction $\mathrm{z}$ as well as in the vertical direction $\mathrm{y}$. The $5.7 \mathrm{~cm}$ pitch of the probes in the third dimension $\mathrm{x}$ is determined by the precisely machined fixing points of the cards on the arms of the mapping device.

The main aim of the mapping campaign was a detailed field map of the ND280 TPC region at a current of 1000 A equivalent to a field of $\sim 712 \mathrm{G}$ in the center of the magnet. The map consists of more than 250,000 measurement points which corresponds to a $5 \mathrm{~cm}$ distance of measurements in $y$ - and z-direction. The procedure for the calibration of this data is discussed in this section.

For the equalization of the probes in the ND280 magnet an offset correction was applied. This offset $c_{0}$ is determined from the data of all probes by applying the following fitting function to the main B-field component:

$$
B[G]=c_{0}+c_{1} I+c_{1} c_{2} I^{2}
$$

The transverse B-field components $B_{y}$ and $B_{z}$ were not considered to retrieve the fitting parameters $c_{1}$ and $c_{2}$, since the amplitude of these field components is so small that the error is dominated by the intrinsic resolution of the Hall probes (0.2 Gauss).

The purpose of the calibration procedure is to determine the fitting parameters $c_{0}, c_{1}$ and $c_{2}$ of eq. (3.1). The $c_{2}$ parameter can be considered to depend solely on the magnetization fetaures of the iron yoke. In fact, it also takes into account the magnetization of all surrounding materials such as the basket or the mapping device itself, but the influence of those can be regarded as negligible. The mean value of $c_{2}$ is obtained from the fits at each measurement point, and with this given value of $c_{2}$ the fit is then repeated for each probe and each direction $\left(B_{x}, B_{y}\right.$ and $\left.B_{z}\right)$. The remaining two parameters $c_{1}$ and $c_{0}$ describe the strength of the field and the offset value for each probe, respectively. A distribution of the obtained offsets is shown in figure 10.

Systematic effects of the mapping device geometry include the skewing of the mapping device itself and angular misalignments between the Hall probes. The data was corrected by measuring these effects and taking them into acount. When moving the device in the z-direction the two pneumatic motors are individually controlled to ensure a smooth movement along the rails. This feature allowed for a better control of the movement and avoidance of mechanical stress on the equipment. The two motors, separated by $d_{z}=2.2 \mathrm{~m}$, may stop at slightly different positions in the z-direction for each measurement point $\left(\Delta z=z_{1}-z_{0}<1 \mathrm{~mm}\right)$. This introduces a systematic skewing of the device with an angle below $0.5 \mathrm{mrad}$, and we correct the angular alignment of the mapping device accordingly. As mentioned in section 2.1, optical encoders measure the stopping positions of both sides of the device. The data are used to determine the skewing angles. Additional skewing was observed in y which exhibited dependence on both y position and direction of movement and was found to be due to friction and mechanical stress on the device. The displacement $\Delta y$ was taken from two encoders on the y-axis of the device, separated by $d_{y}$. The angles for the skewing $\delta_{i}$ are 

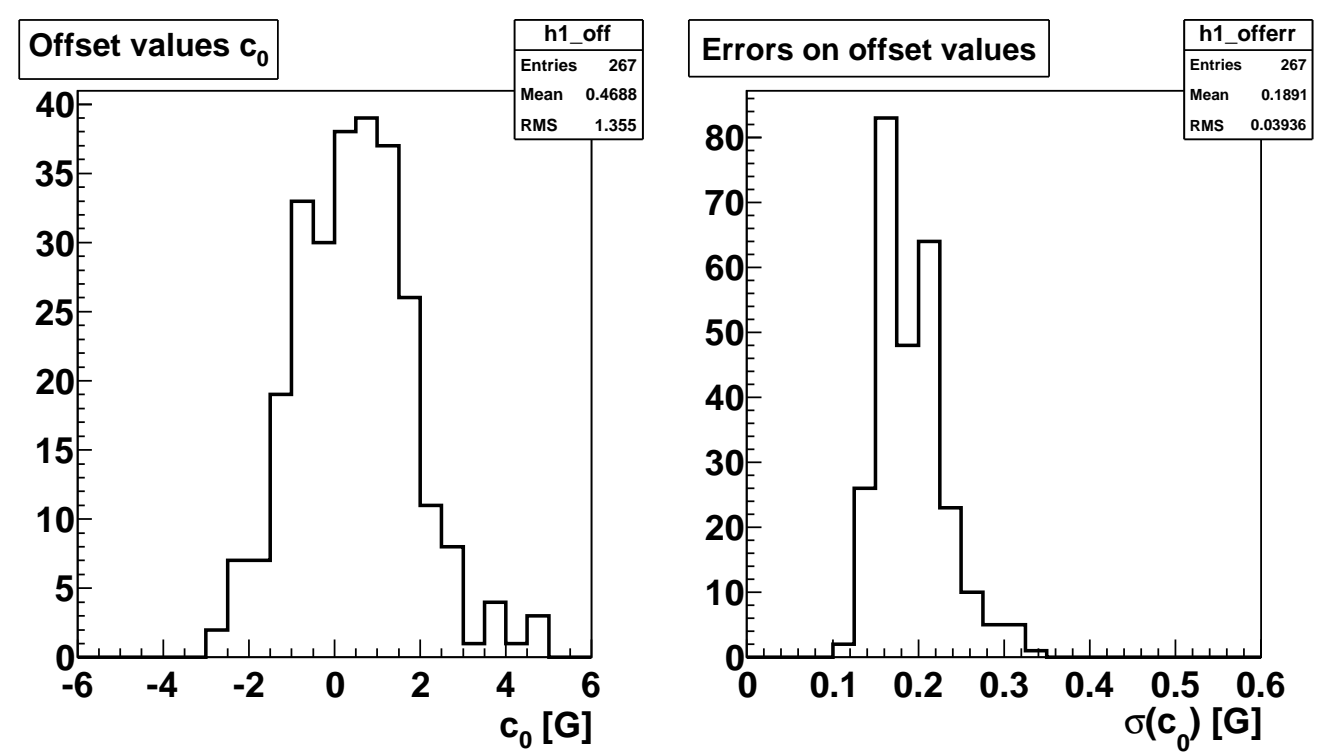

Figure 10. Distribution of the fitted $c_{0}$ parameter (left plot) and its error (right plot) for each probe.

small being of the order of a mrad, therefore, we obtain the following equations:

$$
\begin{aligned}
& \sin \delta_{y} \approx \tan \delta_{z}=\Delta y / d_{y}, \quad \sin \delta_{z} \approx \tan \delta_{z}=\Delta z / d_{z} \\
& B_{x}^{\prime}=B_{x}\left(\cos \delta_{y}+\cos \delta_{z}\right)-B_{y} \sin \delta_{y}-B_{z} \sin \delta_{z} \approx B_{x} \\
& B_{y}^{\prime}=B_{y} \cos \delta_{y}+B_{x} \sin \delta_{y} \approx B_{x} \sin \delta_{y} \\
& B_{z}^{\prime}=B_{z} \cos \delta_{z}+B_{x} \sin \delta_{z} \approx B_{x} \sin \delta_{z},
\end{aligned}
$$

The corrected values $B_{i}^{\prime}$ are used for further analysis. As an example, figure 11 shows the effect of the correction for $B_{y}$. With this method one can correct for effects at the level of the intrinsic probe uncertainty of 0.2 Gauss.

The misalignment of each probe with respect to each other must be taken into account as well. From the calibration procedure (section 2.3) it is known that the probes should agree within $\pm 2 \mathrm{mrad}$. For the transverse components $B_{y}$ or $B_{z}$, the alignment can be further refined by exploiting the cartesian symmetry of the magnet geometry and the fact that the center of the magnet is defined as the origin of the ND280 coordinate system. In the horizontal symmetry plane of the coils at $y=0$, we expect the vertical field distortions for $B_{y}$ to vanish. An analogue statement holds for the $B_{z}$ component.

In these planes of symmetry ( $y=0$ or $z=0$ ) either $\left|B_{y}\right|$ or $\left|B_{z}\right|$ should be minimal everywhere. The mean value of one B-field component of the 39 probes along one arm is taken and defined to be the reference value:

$$
\bar{B}_{y}=\frac{1}{39 \cdot n} \sum_{i=1}^{n} \sum_{j=1}^{39} B_{y, i j}
$$

where $n$ is the number of measurement points per probe ( $n$ steps in z-direction for $B_{y}$ ). The measurements of each probe are corrected to equal this mean value obtained from the symmetry planes. In figure 12 the improvement of the data quality for $B_{y}$ in the plane where $y=0$ is shown. For $x=0$, 

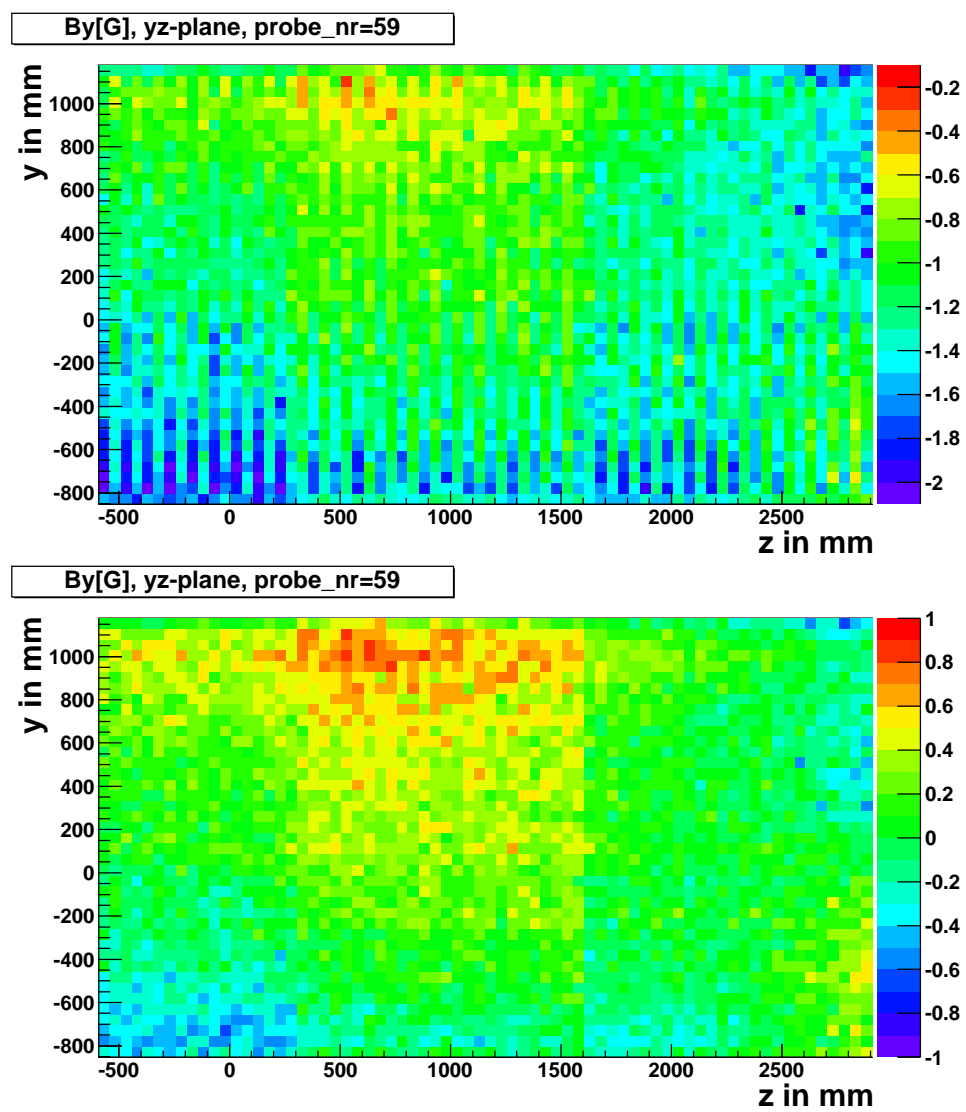

Figure 11. Measurements of the Hall probe at $x=0$ for all positions in $\mathrm{y}$ and $\mathrm{z}$. This corresponds to a side view of the ND280 TPC region. The colors indicate the $B_{y}$ field values in Gauss. An improvement of data quality after correcting for skewing effects of the device is visible (top: without correction; bottom: with correction). The differences between neighboring measurements in z-direction are reduced (columns) and also the mean value of all measurements moves closer to 0 Gauss. The shifts of 0.2 Gauss for $z=300 \mathrm{~mm}$ and $z=1600 \mathrm{~mm}$ occurred after two earth quakes, which might have caused a small shift $(<1 \mathrm{~mm})$ of the mapping equipment with respect to the magnet.

the $B_{x}$ value is not minimal since this is the main component of the field, which is intended to be as large and uniform as possible. Hence, a correction for $B_{x}$ can only be obtained from a fitting procedure which is described in detail in [8].

The overall effect of the equalization and alignment corrections can be seen in figure 13, in which the differences for $B_{y}$ and $B_{z}$ between adjacent probes are shown. With RMS values of 0.17 Gauss and 0.24 Gauss the resulting deviations are compatible with the intrinsic resolution of the Hall probes.

\section{Conclusions}

A dedicated, novel device was built to map the magnetic field of the magnet of the ND280 detector complex of the T2K neutrino experiment. The mapping device was exclusively made of non- 


\section{By[G], xz-plane, arm2, y=0}

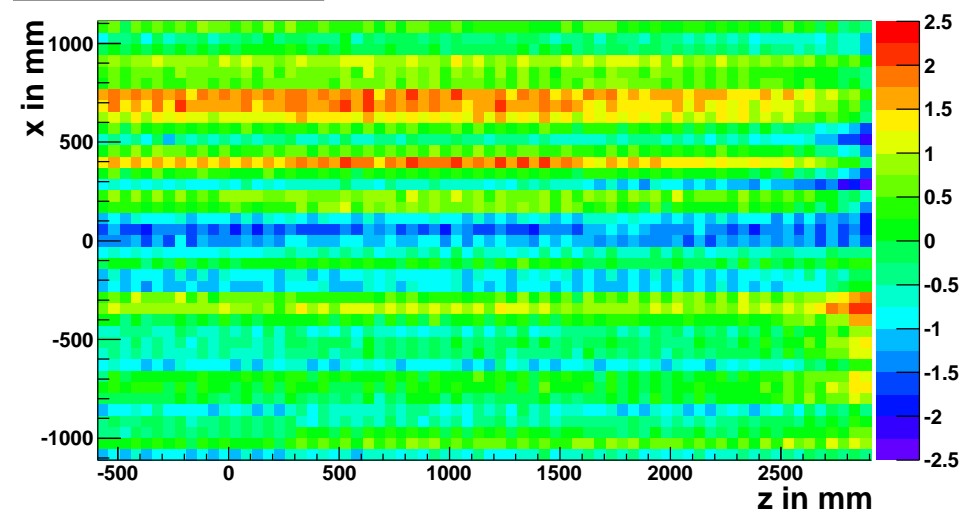

By[G], xz-plane, arm2, y=0

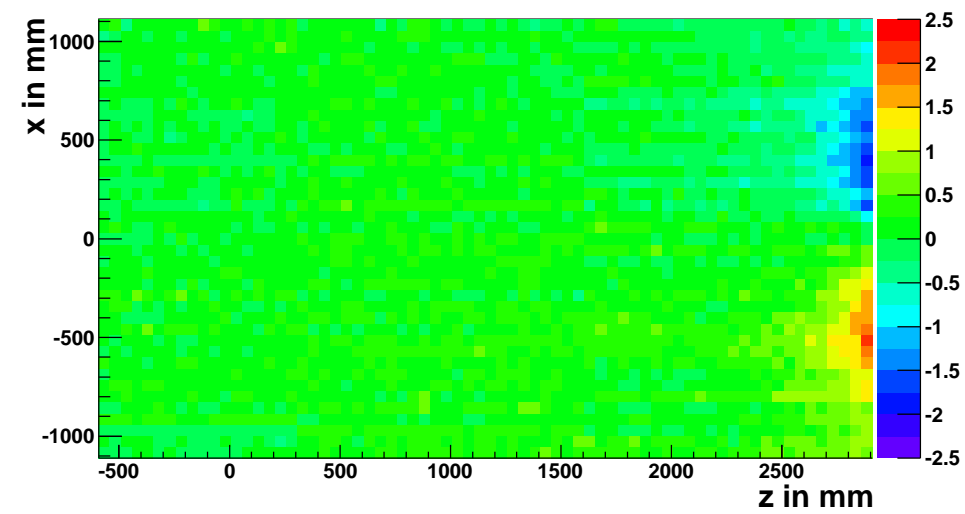

Figure 12. Top view of the ND280 TPC region. The colors represent the $B_{y}$ field values in Gauss. Each row corresponds to the measurements of one probe. The variation of the values between the probes vanishes when applying the corrections described in the text (top: without correction; bottom: with correction). The blue and red colored regions on the right-hand-side of the bottom plot show the influence of the coils. This influence can be determined with sufficient accuracy only after the corrections.

magnetic parts and completed with a set of high precision Hall probes. The absolute scale of the magnetic field is ensured by a careful calibration of each Hall probe in a reference magnetic field.

With the help of a dedicated mapping campaign it is demonstrated that the required measurement accuracy of the order of a Gauss is achieved. This meets the requirements for the momentum accuracy for particles with the use of the ND280 TPCs.

\section{Acknowledgments}

We thank all colleagues who helped to design and construct the mapping device and to ensure smooth measurements. Special thanks go to the T2K magnet group for providing many suggestions and a lot of support in operating the magnet, and also to Dean Karlen and the CERN survey group for their efforts in surveying the equipment. 


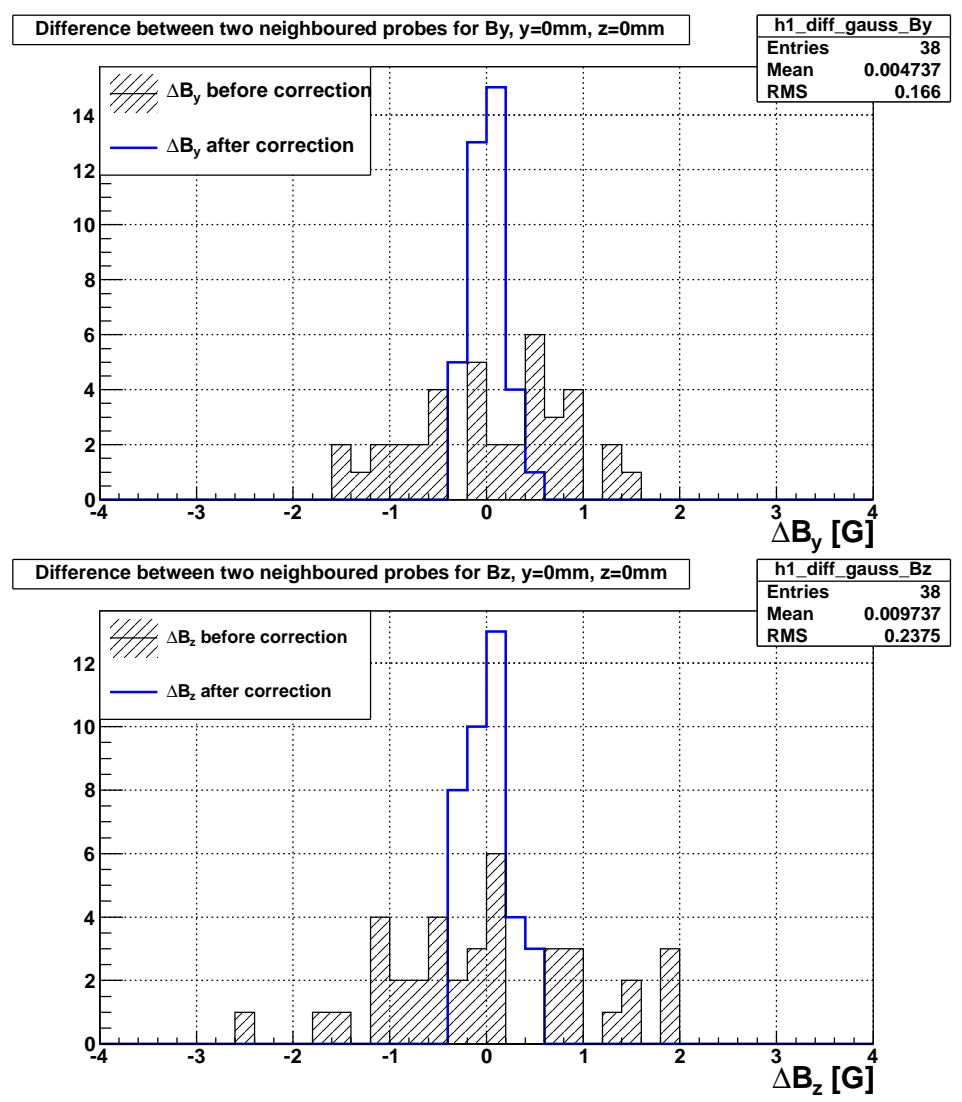

Figure 13. Deviations of the measured values between adjacent probes before (shaded black) and after the corrections (blue line) for $B_{y}$ (top) and $B_{z}$ (bottom). The fact that the distributions center around zero shows that there is no rotational bias along the $\mathrm{x}$-direction.

\section{References}

[1] T2K collaboration, Y. Itow et al., The JHF-Kamioka neutrino project, hep-ex/0106019.

[2] T2K collaboration, K. Abe and J.B. Spitz, The T2K experiment, Nucl. Instrum. Meth. A 659 (2011) 106 [arXiv:1106.1238].

[3] T2K collaboration, K. Abe et al., Indication of electron neutrino appearance from an accelerator-produced off-axis muon neutrino beam, Phys. Rev. Lett. 107 (2011) 041801 [arXiv:1106.2822].

[4] B. Pontecorvo, Mesonium and antimesonium, Zh. Eksp. Teor. Fiz. 33 (1957) 549 [Sov. Phys. JETP 6 (1957) 429]; Inverse beta processes and nonconservation of lepton charge, Zh. Eksp. Teor. Fiz. 34 (1958) 247 [Sov. Phys. JETP 7 (1958) 172];

Z. Maki, M. Nakagawa and S. Sakata, Remarks on the unified model of elementary particles, Prog. Theor. Phys. 28 (1962) 870.

[5] UA1 collaboration, G. Arnison et al., Experimental observation of lepton pairs of invariant mass around $95 \mathrm{GeV} / \mathrm{c}^{2}$ at the CERN SPS collider, Phys. Lett. B 126 (1983) 398. 
[6] NOMAD collaboration, J. Altegoer et al., The NOMAD experiment at the CERN SPS, Nucl. Instrum. Meth. A 404 (1998) 96.

[7] T2K ND280 TPC collaboration, N. Abgrall et al., Time Projection Chambers for the T2K near detectors, Nucl. Instrum. Meth. A 637 (2011) 25 [arXiv: 1012.0865].

[8] E. Frank, Precision measurement of the ND280 magnetic field in the T2K neutrino experiment: confirmation of the momentum scale through $\Delta^{++}$resonances, Ph.D. thesis, in preparation.

[9] T2K ND280 MAGNET collaboration, Installation, calibration and operation of a large dipole magnet for the ND280 off-axis detector of the T2K experiment, in preparation.

[10] Hall probes developed by NIKHEF webpage. http://www.nikhef.nl/pub/departments/ct/po/html/dcs.html.

[11] Robert Bosch GmbH, CAN - Controller Area Network funktionelle Beschreibung (in German), Germany (1987).

[12] D. Karlen, Aligning the tracker in the ND280 basket, T2K technical note T2K-TN-010, http://www.t2k.org/docs/technotes/010/tn010/view.

[13] F. Bergsma, Calibration of Hall sensors in three dimensions, in proceedings of $13^{\text {th }}$ International Magnetic Measurement Workshop, SLAC, U.S.A. (2003).

[14] M. Aleksa et al., Measurement of the ATLAS solenoid magnetic field, 2008 JINST 3 P04003.

[15] M. Aleksa et al., Measurement of the solenoid magnetic field, ATLAS note ATL-MAGNET-PUB-2007-001, CERN, Geneva Switzerland (2007). 